Di sper si on anal ysi s of crack-waves i $n$ an artificial subsurface fracture using two crack model s

\begin{tabular}{|c|c|}
\hline 著者 & NAGANO Koj i, N I TSUMA Hi r oaki \\
\hline $\begin{array}{l}\text { jour nal or } \\
\text { publ i cat i on } \mathrm{titl} \text { e }\end{array}$ & $\begin{array}{l}\text { I EEE transact i ons on geosci ence and rente } \mathrm{e} \\
\text { sensi ng }\end{array}$ \\
\hline vol une & 38 \\
\hline nunber & 1 \\
\hline page $r$ ange & 311 \\
\hline year & 2000-01 \\
\hline URL & ht t p: //hdl . handl e. net /10258/200 \\
\hline
\end{tabular}


Di sper si on anal ysi s of crack-waves i $n$ an artificial subsurface fracture using two crack model s

\begin{tabular}{|c|c|}
\hline 著者 & NAGANO Koj i, N I TSUMA Hi r oaki \\
\hline $\begin{array}{l}\text { jour nal or } \\
\text { publ i cat i on } \mathrm{titl} \text { e }\end{array}$ & $\begin{array}{l}\text { I EEE transact i ons on geosci ence and rente } \mathrm{e} \\
\text { sensi ng }\end{array}$ \\
\hline vol une & 38 \\
\hline nunber & 1 \\
\hline page $r$ ange & 311 \\
\hline year & 2000-01 \\
\hline URL & ht t p: //hdl . handl e. net /10258/200 \\
\hline
\end{tabular}




\title{
Dispersion Analysis of Crack-Waves in an Artificial Subsurface Fracture Using Two Crack Models
}

\author{
Koji Nagano and Hiroaki Niitsuma
}

\begin{abstract}
We investigated crack-wave dispersions in an artificial subsurface fracture both experimentally and numerically using a wavelet analysis and two crack models. Crack-waves are seismic modes that propagate along a fracture. The dispersion characteristics of crack-waves depend on the geometry and physical properties of a fracture. We measured crack-waves at an artificial subsurface fracture in Higashi-Hachimantai Hot Dry Rock model field, Japan. This subsurface fracture is at a depth of about $370 \mathrm{~m}$. During a measurement, we injected water into the fracture and changed the interface conditions of the fracture. A wavelet analysis provided the dispersion of the arrival times of crack-waves. The crack-waves showed positive velocity dispersion; i.e., low frequency components arrived later. As wellhead pressure increased due to water injection, the dispersion characteristics changed. A low-velocity-layer (LVL) model and a crack-stiffness model were examined to explain crack-wave dispersion. In the LVL model, rock layers with a low velocity surround a fluid layer. There is no contact between the LVL's. On the other hand, the crack-stiffness model considers crack stiffness due to contact between asperities on fracture surfaces. The arrival-time curves calculated by the crack-stiffness model showed a good fit to the measured values. As wellhead pressure increased, crack stiffness decreased and thickness of a fluid layer increased. In contrast, the LVL model did not adequately duplicate the measured data.
\end{abstract}

Index Terms-Crack, crack-waves, dispersion, wavelet.

\section{INTRODUCTION}

A FLUID-FILLED crack and a fracture zone with low velocities act as elastic interfaces for seismic waves. Such interfaces trap seismic waves, and trapped seismic waves propagate along the interfaces. They are referred to as crack-waves, crack-interface waves, and fault zone-guided waves [1]-[3]. In this paper, we refer to these seismic modes as crack-waves. The propagation characteristics of crack-waves are different from those of reflected or refracted seismic waves, which are used in conventional seismic surveys. Their waveforms (e.g., velocity-frequency dispersion and amplitude-space distribution) are strongly dependent on the crack's geometry and its physical properties. Therefore, measurement of crack-waves is an effective tool for characterizing a subsurface fracture.

Manuscript received July 1, 1998; revised March 24, 1999. This work was supported by a Grant-in Aid for Encouragement of Young Scientists, Ministry of Education, Science, and Culture of Japan under Contracts 08751081 and 10750667 and the Espec Foundation for Earth Environmental Research and Technologies (Charitable Trust), and was conducted as part of the MTC (More Than Cloud) international collaborative project funded by NEDO, Japan.

K. Nagano is with the Department of Computer Science and Systems Engineering, Muroran Institute of Technology, Muroran 050-8585, Japan (e-mail: nagano@csse.muroran-it.ac.jp).

H. Niitsuma is with the Department of Geoscience and Technology, Graduate School of Engineering, Tohoku University, Sendai 980-8579, Japan (e-mail: niitsuma@ni4.earth.tohoku.ac.jp).

Publisher Item Identifier S 0196-2892(00)00001-2.
We previously studied the propagation of crack-waves to characterize subsurface fractures [4]-[7]. We detected crack-waves propagating along an artificial subsurface fracture, which was saturated with water at a depth of $370 \mathrm{~m}$ in Higashi-Hachimantai Hot Dry Rock model field, Japan [4]. We estimated the dimensionless crack stiffness of the fracture surfaces based on dispersions of the crack-waves [5], [8]. In this analysis, we applied a pseudo-Wigner-Ville distribution (PWD) to estimate the dispersions of crack-waves. However, it was difficult to estimate dispersions because of cross terms in the PWD. Cross terms naturally occur in energy densities of the PWD. The dimensionless crack stiffness is calculated from three physical properties of a fracture (thickness of the fluid layer, crack stiffness, and the shear modulus of the solid). We could not estimate the primary physical properties of a fracture in the crack-wave analysis, because the dimensionless crack stiffness is a theoretical parameter.

Niitsuma and Saito [9] and Tanaka et al. [10] reported that a low-velocity zone could be found close to an artificial subsurface fracture that had been created in an intact rock layer by hydraulic fracturing. The velocity of a compressional wave decreased in a path through the fracture when wellhead pressure increased due to water injection into the fracture. There was no natural crack in the rock layer before hydraulic fracturing. They explained this decrease in compressional wave velocity in terms of the reopening of microcracks in the vicinity of the fracture. This phenomenon indicates that a low-velocity zone is essential for subsurface fractures. Therefore, it is important to estimate the low-velocity zone when subsurface fractures are analyzed.

The numerical analysis of crack-waves trapped in a single fluid-filled crack has been reported by Chouet [1], Ferrazzini and Aki [11], and Hayashi and Sato [8]. All of these authors addressed very slow waves with positive dispersion. Chouet, and Ferrazzini and Aki used a simple three-layer model for a subsurface crack. A very thin fluid layer, compared with the wavelength, was sandwiched between two solid half-spaces. Hayashi and Sato focused on the contact between asperities on fracture surfaces [8]. The dimensionless crack stiffness was used to represent contact in their crack model.

In this study, we investigate the opening of a subsurface fracture by comparing the dispersions of crack-waves measured in the field and numerical results calculated using two crack models. The crack-waves were measured at an artificial subsurface fracture at a depth of about $370 \mathrm{~m}$. Two waveforms of the crack-waves, which were recorded at the beginning of water injection and at the maximum wellhead pressure during water injection, are analyzed. The wavelet transform (WT) provides a time-frequency representation of the arrival time of 


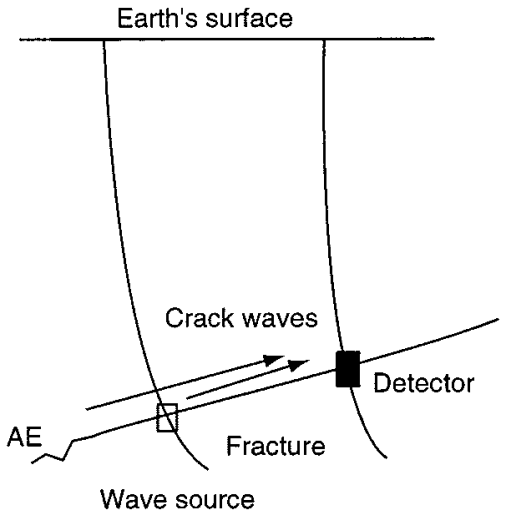

Fig. 1. Concept of crack-wave measurement.

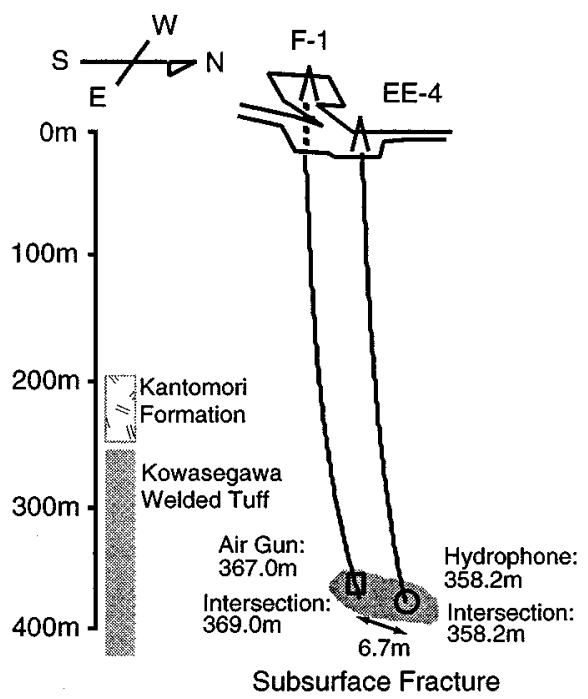

Fig. 2. Higashi-Hachimantai HDR model field. Wells EE-4 and F-1 intersect an artificial subsurface fracture at depths of 358.2 and $369.0 \mathrm{~m}$, respectively.

crack-waves. The two crack models are the crack-stiffness model, which was investigated by Hayashi and Sato [8], and the low-velocity layer (LVL) model, which is a modified three-layer model based on the experimental results of Niitsuma and Saito [9]. We calculate the arrival time curves of crack-waves with the two crack models and fit these curves to the spectral data in the time-frequency representation from the WT. We estimate the physical parameters of the crack models in the opening of a fracture based on crack-wave dispersions.

\section{CRaCK-WaVe Measurement In Higashi-Hachimantai HOT DRY ROCK (HDR) MODEL FIELD}

Crack-waves were measured at Higashi-Hachimantai Hot Dry Rock (HDR) model field, Japan [12]. Fig. 1 illustrates the concept of a crack-wave measurement. Crack-waves can be detected at the intersection of a bore hole and the fracture. Fig. 2 depicts Higashi-Hachimantai HDR model field. An artificial subsurface fracture was created in intact, welded tuff at a depth of $369.0 \mathrm{~m}$ in well F-1 by hydraulic fracturing. During hydraulic fracturing, 40-mesh sand was injected as a propping agent. Core samples of well F-1 showed no significant joint or crack before the fracturing. Well EE-4 was drilled

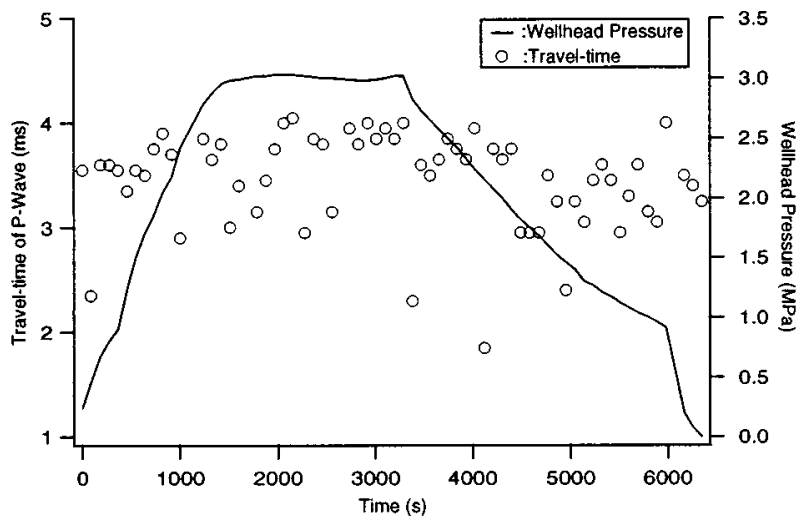

Fig. 3. Wellhead pressure and travel time of the compressional wave during water injection.

into the artificial fracture after the hydraulic fracturing and intersected the artificial fracture at a depth of $358.2 \mathrm{~m}$. The distance between the intersection points of wells F-1 and EE-4 was $6.7 \mathrm{~m}$. The radius of the fracture was about $60 \mathrm{~m} \mathrm{[12].} \mathrm{A}$ transmissibility test showed that the fracture aperture was about $0.08 \mathrm{~mm}$ without pressurization and $0.2 \mathrm{~mm}$ at a wellhead pressure of 3.0 MPa [13]. The transmissibility test also showed that the reopening pressure of this fracture system was about 2.8 MPa at the wellhead. The velocities of compressional and shear waves of intact rock in this field are $3100 \mathrm{~m} / \mathrm{s}$ and 1860 $\mathrm{m} / \mathrm{s}$, respectively [10].

We carried out our measurements using this single artificial fracture. A downhole air gun was used as a wave source at a depth of $367.0 \mathrm{~m}$ in well F-1. The air gun was suspended two meters from the intersection of the fracture so that we would not damage the bore hole at the intersection. A hydrophone was suspended at a depth of $358.2 \mathrm{~m}$ in well EE-4 (i.e., at the intersection with the fracture), to measure the crack-waves. This hydrophone has a flat sensitivity in a frequency range of $60 \mathrm{~Hz}$ to $10 \mathrm{kHz}$, and its sensitivity gradually decreases for frequency components below $60 \mathrm{~Hz}$.

We pressurized the subsurface fracture to vary the interface conditions of the fracture. The wellheads of both wells were closed with wireline lubricators. Wellhead pressure was measured in well F-1. Since circulation loss and friction loss in the bore holes and this fracture system are small, the wellhead pressure in well EE-4 is almost equal to that in well F-1 [12]. Fig. 3 shows the wellhead pressure during crack-wave measurement. After we held the wellhead pressure at $3.0 \mathrm{MPa}$ for $30 \mathrm{~min}$, we closed the wellheads completely. Wellhead pressure subsequently decreased naturally due to permeation into the rock.

Fig. 3 also shows the travel time of a compressional wave in the crack-wave measurement. Since the air gun was two meters above the intersection of the bore hole and the hydrophone was at another intersection, the path of the compressional wave was close to the fracture. The velocity of the compressional wave was calculated from the travel time and the distance, $6.7 \mathrm{~m}$, between the intersections of the bore holes and the fracture. The velocity was $1900 \mathrm{~m} / \mathrm{s}$ at the beginning of water injection and $1700 \mathrm{~m} / \mathrm{s}$ when the wellhead pressure was constant at $3.0 \mathrm{MPa}$ for about $30 \mathrm{~min}$. These velocities are lower than that measured in other experiments in this field [10]. Another water-injection 


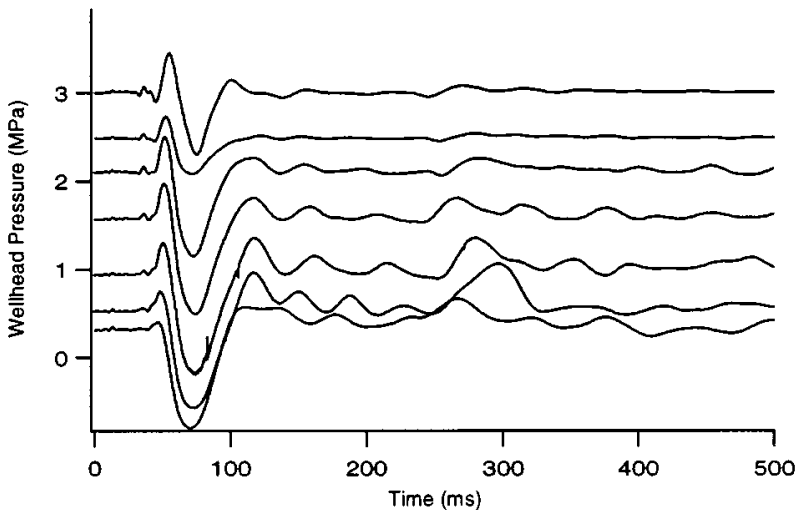

Fig. 4. Crack-waves during water injection in an artificial subsurface fracture The crack-waves arrive at $40 \mathrm{~ms}$.

test was carried out about one hour before the crack-wave measurement. These lower velocities indicate that the rock near the fracture was undergoing relaxation after the previous water-injection.

Fig. 4 shows waveforms detected during water injection. The crack-waves convert to tube waves at the intersection with a bore hole. Tube-wave analysis indicated that the waves with a large amplitude at $40 \mathrm{~ms}$ in Fig. 4 are crack-waves [4]. As the wellhead pressure was increased, the waveforms of the crackwaves changed.

\section{WAVELET ANALYSIS OF CRACK-WAVES}

We used the wavelet transform (WT) to obtain spectral data as a function of arrival time in a dispersion analysis of the crackwaves [14]-[16]. Time and frequency resolutions vary in the WT, while they are uniform in the traditional short-time Fourier transform (STFT). This variable resolution is an advantage of the WT over the STFT. The WT is defined as

$$
W_{\phi}(a, b)=\frac{1}{\sqrt{a}} \int_{-\infty}^{\infty} \phi^{*}\left(\frac{t^{\prime}-b}{a}\right) s\left(t^{\prime}\right) d t^{\prime}
$$

where $\phi(t)$ is the analyzing wavelet, $a$ is a scale parameter, $b$ is a time-shift parameter, and * denotes the complex conjugate. The scale parameter $a$ is the reciprocal of the frequency. The analyzing wavelet requires the admissibility condition given by

$$
c_{\phi}=2 \pi \int_{-\infty}^{\infty} \frac{|\hat{\phi}(\omega)|^{2}}{|\omega|} d \omega<\infty
$$

where $\hat{\phi}$ is a Fourier transform of the analyzing wavelet $\phi$. The analyzing wavelet in this paper is the modulated Gaussian $\phi_{\mathrm{M}}(t)$ given by

$$
\phi_{\mathrm{M}}(t)=\exp \left(-\frac{t^{2}}{2}+j m t\right) .
$$

When the analyzing wavelet is the modulated Gaussian, uncertainty in the time-frequency domain is minimum. Therefore, the modulated Gaussian is efficient for representing a spectral component that varies with time. The modulated Gaussian does not strictly satisfy the admissibility condition. However, if $m>5$ in (3), we can consider that the admissibility condition is satisfied [17]. In the WT calculation, we set $m=6$.

We analyzed the WT of the crack-waves in a frequency range between 2 and $256 \mathrm{~Hz}$ because of the frequency components of the air gun. The waveforms and squared amplitudes of the WT are presented in Fig. 5. Fig. 5(a) shows the crack-waves detected at the beginning of water injection. The wellhead pressure is $0.4 \mathrm{MPa}$. The crack-waves in Fig. 5(b) were detected when the wellhead pressure was held constant at 3.0 MPa for about $30 \mathrm{~min}$. The squared amplitude of the WT was normalized by its maximum value at each frequency to enhance the contrast of the variation in time for frequency components with small amplitude. The air gun exploded seismic waves at a frequency range below $300 \mathrm{~Hz}$ simultaneously [5]. Crack-waves are dispersive, since arrival time (which occurs at the onset of the relative maxima) is a function of frequency in Fig. 5. The lower frequency components of the crack-waves arrive later. The velocity dispersion at a wellhead pressure of $0.4 \mathrm{MPa}$ is weaker than that at a wellhead pressure of 3.0 $\mathrm{MPa}$.

\section{CRACK MODELS}

A basic crack model for a fluid-filled crack is a three-layer model, as discussed by Ferrazzini and Aki [11], in which a thin fluid layer, compared with the wavelength of the crack-waves, is sandwiched between two solid half-spaces. There is no contact between the two solids. The three-layer model is not valid for the analysis of crack-waves propagating along an artificial subsurface fracture. In particular, since a low-velocity zone was detected even in the vicinity of a fracture created in an intact rock layer [9], [10], a low-velocity zone should be taken into account in an analysis of subsurface fractures. Contact between asperities on fracture surfaces is also inherent in subsurface fracture.

We examined crack-wave dispersions using two crack models: the LVL model and the crack-stiffness model. The two physical properties, a low-velocity zone and contact, are independently introduced into a three-layer model. These crack models, which are independent of each other, demonstrate the effects of these physical properties of a fracture.

Dispersion equations for the LVL and crack-stiffness models are derived using common potential functions for the wave equation. We assume that layers lie in the $x y$-plane and that the normal to the wavefront lies on the $x$ axis. Periodic solutions of the wave equation may be found by combining with a compressional wave solution

$$
\phi_{m}=\left(\Delta_{m}^{\prime} e^{-j \xi r_{\alpha m} z}+\Delta_{m}^{\prime \prime} e^{j \xi r_{\alpha m} z}\right) e^{j(\omega t-\xi x)}
$$

and a shear wave solution

$$
\psi_{m}=\left(\Omega_{m}^{\prime} e^{-j \xi r_{\beta m} z}+\Omega_{m}^{\prime \prime} e^{j \xi r_{\beta m} z}\right) e^{j(\omega t-\xi x)}
$$

where $\omega$ is the angular frequency, $\xi$ is the wavenumber, and $\Delta_{m}^{\prime}, \Delta_{m}^{\prime \prime}, \Omega_{m}^{\prime}$, and $\Omega_{m}^{\prime \prime}$ are unknown functions of $\omega$ and $\xi$. Subscript $m$ denotes the number of a layer.

Higher order modes show more oscillation of pressure along the $z$ axis in a fluid layer. The aperture of a fluid layer is quite narrow in this analysis. Therefore, higher order modes have much less energy than the fundamental mode. The numerical 

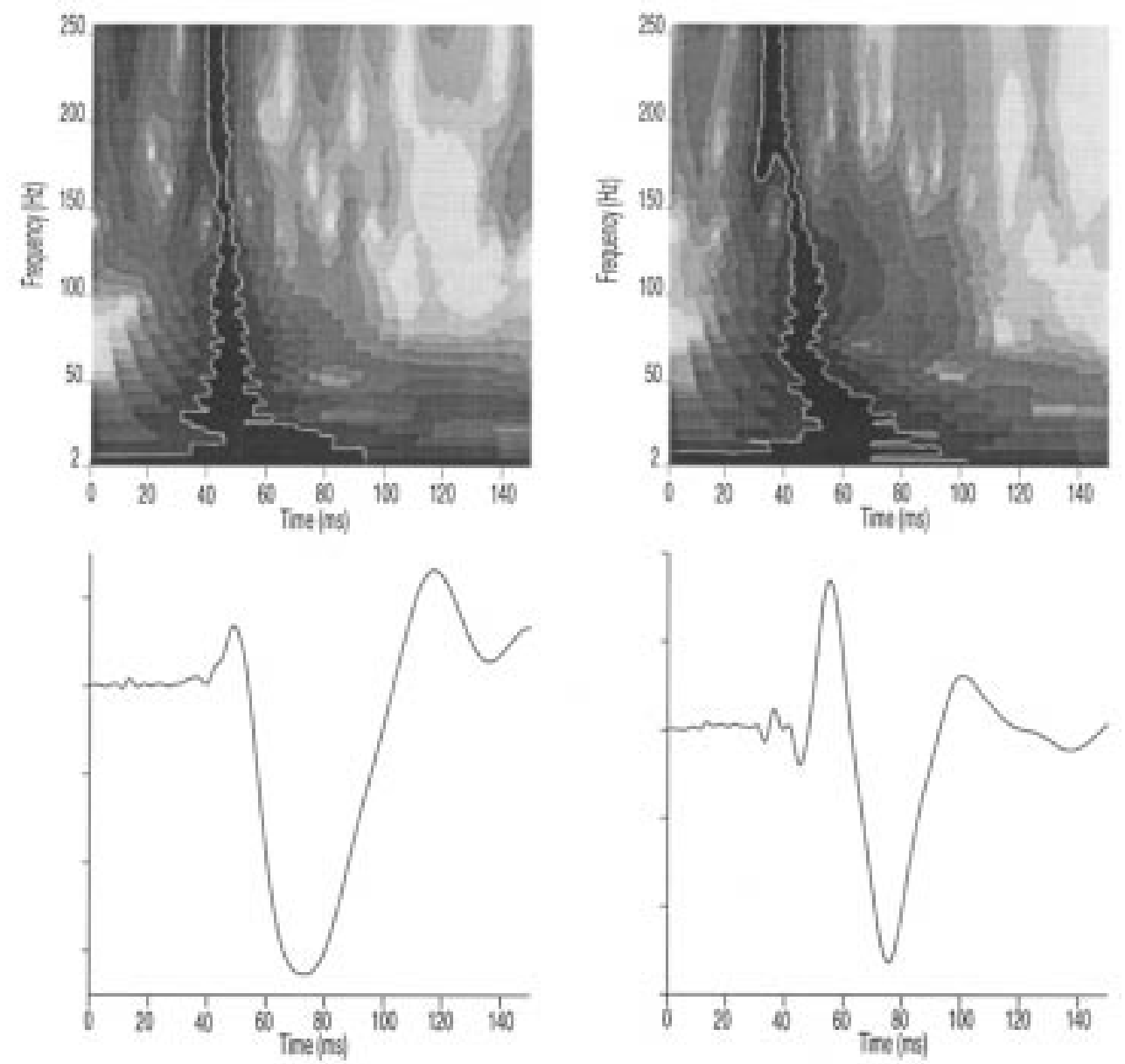

(a)

(b)

Fig. 5. Wavelet transforms of the crack-waves. The arrival time of each frequency component can be seen. (a) The crack-wave detected at a wellhead pressure of 0.4 MPa. These data were recorded at the beginning of the injection. (b) The crack-wave detected when the wellhead pressure was held constant at 3.0 MPa for $1779 \mathrm{~s}$ (about $30 \mathrm{~min}$ ). The aperture of the subsurface fracture increases when the wellhead pressure approaches 3.0 MPa.

analysis of Ferrazzini and Aki [11] showed that only the fundamental symmetric mode of crack-waves was strongly affected by the interface conditions at fracture surfaces. Therefore, in this paper, we analyzed the fundamental symmetric modes. Since the velocities of fundamental symmetric modes of crack-waves are lower than the fluid velocity, in the above potential functions

$$
\begin{aligned}
& r_{\alpha m}=-j \sqrt{1-\left(\frac{v}{\alpha_{m}}\right)^{2}}, \\
& r_{\beta m}=-j \sqrt{1-\left(\frac{v}{\beta_{m}}\right)^{2}}
\end{aligned}
$$

where $v$ is the phase velocity of crack-waves, and $\alpha_{m}$ and $\beta_{m}$ are the velocities of compressional and shear waves of the $m$ th layer.

\section{A. Low-Velocity Layer (LVL) Model}

Fig. 6 schematically presents the LVL model. Additional solid layers are found on both sides of the fluid layer. The velocities of these two solid layers are lower than those of the top and bottom bedrock layers. The low-velocity layer (LVL) represents a micro crack zone that is created by pressurization caused by water injection. We assume coordinates to use the

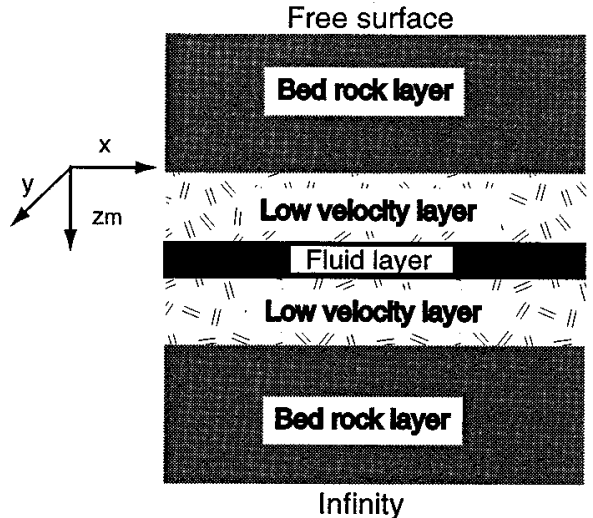

Fig. 6. Low-velocity layer model for an artificial subsurface fracture.

matrix method by Haskell [18]. The top solid layer has a free surface on one side and the bottom solid layer is an infinite half-space. The $x$ axis is parallel to the layers in the direction of propagation. The origin of the $z$ axis is the top surface of each layer. The $z$ axis is positive in the direction of the medium. The layers are numbered starting from the top solid layer. The thickness of the $m$ th layer is $d_{m}$, and the boundaries of the $m$ th layer are at $z_{m}=0$ and $d_{m}$. The $m$ th layer is characterized by 


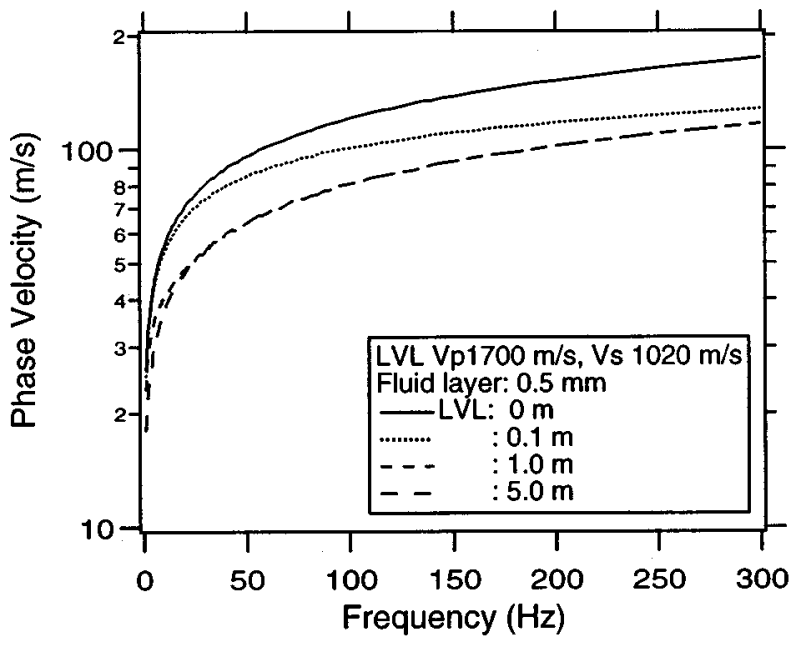

(a)

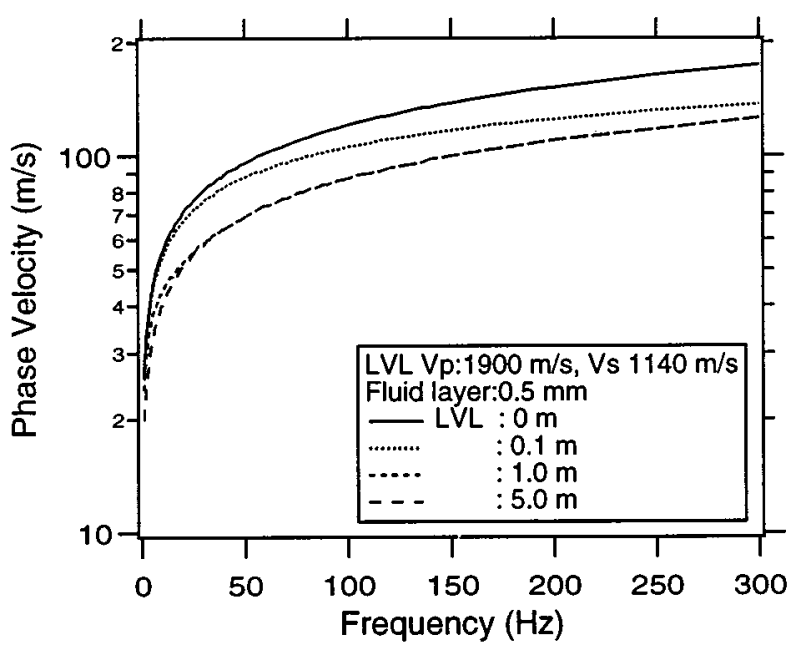

(b)

Fig. 7. Effects of the thickness of the low-velocity layer on the phase velocity of crack-waves: (a) the compressional wave velocity in the low-velocity layer is $1700 \mathrm{~m} / \mathrm{s}$ and (b) the compressional wave velocity in the low-velocity layer is $1900 \mathrm{~m} / \mathrm{s}$.

the compressional wave velocity $\alpha_{m}$, the shear wave velocity $\beta_{m}$, and the density $\rho_{m}$. All of the layers are homogeneous and isotropic, and the fluid is inviscid and incompressible.

At the interface between a bedrock layer and an LVL, normal and shear stresses are continuous, as are displacements in the $x$ and $z$ axes.

At the interface between a fluid layer and an LVL, the normal stress and normal displacement must be continuous and shear stress vanishes. Thus

$$
\left.\begin{array}{rl}
\tau_{z z} & =-\sigma \\
w & =w_{f} \\
\tau_{z x} & =0
\end{array}\right\} \text { at } \begin{aligned}
z_{3} & =0 \\
z_{3} & =d_{3}
\end{aligned}
$$

where $\tau_{z z}$ and $\tau_{z x}$ are the normal and shear stresses, respectively, $\sigma$ is the pressure of the fluid, $w$ is the displacement of the solid in the $z$ axis, and $w_{f}$ is the displacement of the fluid. Since the top surface in the first layer is free, both normal and shear stresses vanish at the top surface. There are no sources at infinity in the bottom layer, so that $\Delta_{5}^{\prime \prime}=\Omega_{5}^{\prime \prime}=0$. We obtain

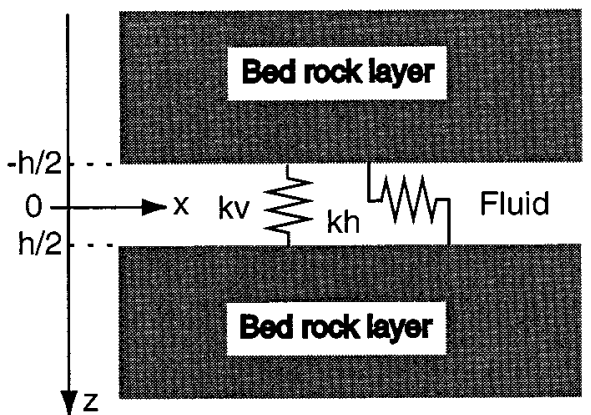

Fig. 8. Crack-stiffness model for an artificial subsurface fracture.

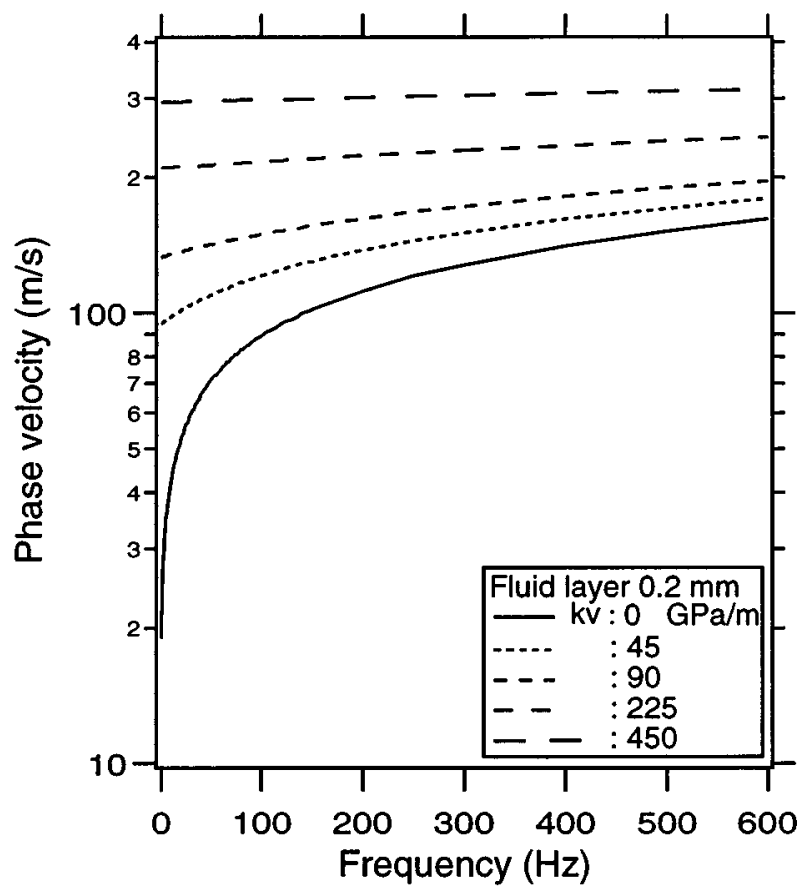

Fig. 9. Dispersion curves of crack-waves in the crack-stiffness model.

the dispersion equation that satisfies these boundary conditions using the matrix method [7], [18].

Fig. 7 shows dispersion curves in the LVL model. When the thickness of an LVL increases, velocities decrease. When the thickness of an LVL exceeds a certain limit, velocities are almost similar in a high frequency range. The limit frequency depends on the thickness of the LVL and the wavelength. For example, in Fig. 7(a), there is little difference between the dispersion curves for the LVL thicknesses of 1.0 and $5.0 \mathrm{~m}$ at a frequency above $30 \mathrm{~Hz}$. It is difficult to measure crack-waves at a very low frequency because of the limited frequency performance of the wave source. Therefore, this convergence means that there is an upper limit for estimating the thickness of an LVL when we measure the velocities of crack-waves.

\section{B. Crack-Stiffness Model}

Fig. 8 shows the crack-stiffness model, in which contact between asperities on fracture surfaces is taken into account [8]. Two solids are connected with springs. A fluid layer is between the solids, and its thickness is $h$. The origin of the $z$ axis is at 


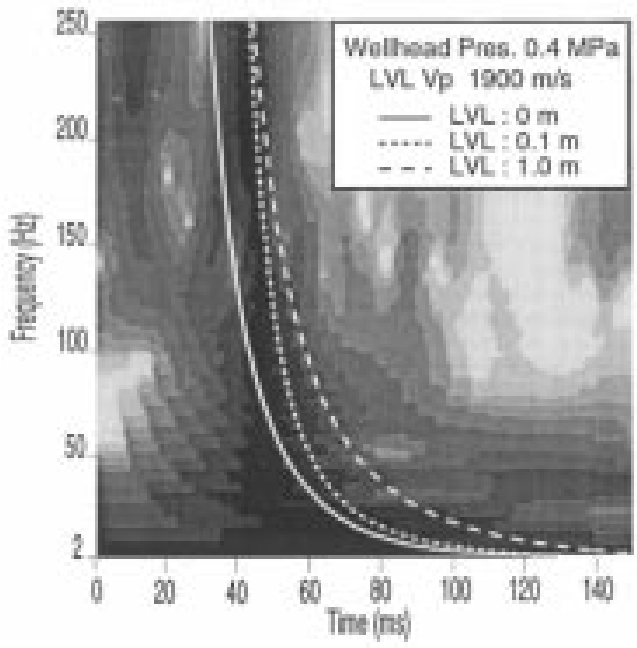

(a)

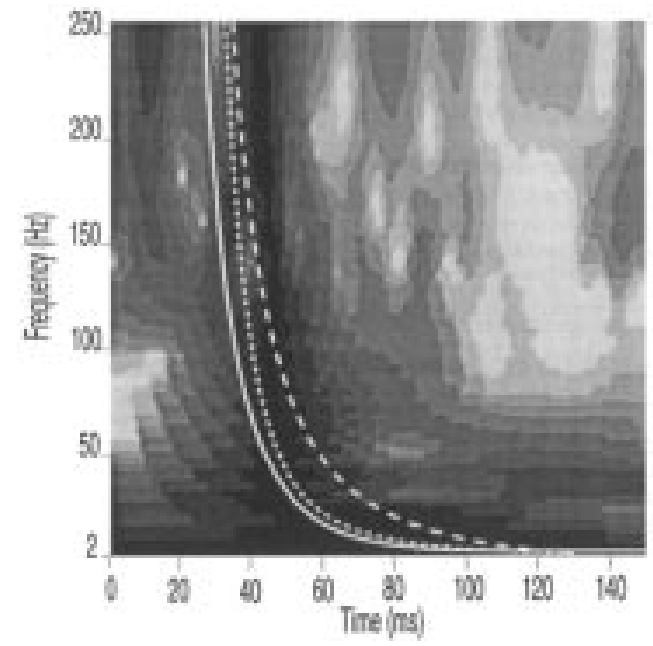

(b)

Fig. 10. Arrival times calculated from group velocities with the LVL model and a time-frequency representation of crack-waves at the beginning of water injection. (a) The fluid layer is $0.5 \mathrm{~mm}$ thick. (b) The fluid layer is $1.0 \mathrm{~mm}$ thick. Compressional and shear wave velocities are 1900 and $1140 \mathrm{~m} / \mathrm{s}$, respectively. The LVL is $0,0.1$, and $1.0 \mathrm{~m}$ thick in (a) and (b).

the center of the fluid layer. The boundary conditions at the interface between a fluid layer and a solid layer are

$$
\left.\begin{array}{rl}
\tau_{z x} & =k_{h}\left(u_{z=h / 2}-u_{z=-(h / 2)}\right) \\
\tau_{z z} & =-\sigma+k_{v}\left(w_{z=h / 2}-w_{z=-(h / 2)}\right) \\
w & =w_{f}
\end{array}\right\} \text { at } z= \pm \frac{h}{2}
$$

where $k_{h}$ and $k_{v}$ are the specific crack stiffnesses in directions parallel and normal to the fluid layer, respectively. The symmetric mode is a function of $k_{h}$, and the antisymmetric mode is a function of $k_{v}$. A dispersion equation is obtained by substituting stresses and displacements derived from (4) and (5) into these boundary conditions [8].

Fig. 9 shows dispersion curves of crack-waves in the crackstiffness model. The thickness of the fluid layer is $0.2 \mathrm{~mm}$, and $k_{v}=0,45,90,225,450 \mathrm{GPa} / \mathrm{m}$ for calculating the dispersion curves in Fig. 9. Crack stiffness increases the phase velocities of crack-waves. Furthermore, the degree of dispersion decreases if crack stiffness increases.

\section{Modeling CRACK-WAVES}

To estimate the physical parameters of these two crack models from the observed crack-waves, we numerically simulate the arrival times of crack-waves for the given crack models. The contours in a scalogram represent the locations of relative maxima of wave energy in the time and frequency domains. A dispersive curve of the relative maxima is observed in the initial motions of the crack-waves in Fig. 5. We fit the arrival times of crack-waves to the dispersive relative maxima in Fig. 5. The arrival times of wave energy are calculated from group velocity and propagation distance. Group velocities are calculated from the phase velocities obtained from dispersion equations in the previous section. Because the hydrophone was suspended at the depth of the intersection with the fracture and the air gun was installed two meters above another intersection with the fracture, we neglect the distance between the air gun and the intersection. The orientation of the artificial fracture, which was estimated from tectonic stress measurement [19], is almost the same as the inclination of the artificial fracture in a core sample, which was obtained from well EE-4. Thus, we assume that the artificial fracture is not curved between the intersections of the bore holes. The propagation distance of crack-waves is $6.7 \mathrm{~m}$ in calculations of the arrival time.

\section{A. Arrival-Time Curves in the LVL Model}

We examined the thicknesses of the fluid layer and the LVL as parameters of the LVL model when calculated arrival times were compared with the dispersion of measured crack-waves. To simplify the calculation, the thicknesses of LVL's are the same on both sides of the fluid layer. We gave typical values for the densities of rock and fluid and fluid velocity. The densities of the bedrock layer and the LVLare $2600 \mathrm{~kg} / \mathrm{m}^{3}$, and the density of the fluid layer is $1000 \mathrm{~kg} / \mathrm{m}^{3}$. Fluid velocity is $1500 \mathrm{~m} / \mathrm{s}$. For other parameters of the LVL model, data measured in the field are used. Velocities of compressional and shear waves in the bedrock layer are 3100 and $1860 \mathrm{~m} / \mathrm{s}$, respectively. The velocity of a compressional wave in the LVL is $1900 \mathrm{~m} / \mathrm{s}$ at the beginning of water injection and 1700 $\mathrm{m} / \mathrm{s}$ at the maximum wellhead pressure. We assume that Poisson's ratio for the LVL is the same as that for the bedrock layer and is constant during waterinjection.

We determined the thicknesses of the fluid layer and the LVL by trial and error. Fig. 10 shows some curves for the calculated arrival times of crack-waves recorded at the beginning of water injection. The background noise level, which exists before the arrival of crack-waves, can be recognized in a contour of the scalogram when we compare the contour to a waveform (Fig. 5). The arrival of a wave occurs at the onset of a relative maximum in a scalogram. The measured crack-waves are less dispersive at a frequency above $30 \mathrm{~Hz}$. On the other hand, calculated arrival-time curves show strong dispersion at frequencies below $70 \mathrm{~Hz}$. Therefore, calculated arrival-time curves are not similar to the dispersion of crack-waves measured at a frequency below $70 \mathrm{~Hz}$. The best combination in our trials was a fluid layer of $1.0 \mathrm{~mm}$ and an LVL of $0.1 \mathrm{~m}$. With this combination, arrival-time curves fit in the frequency range of $70-256 \mathrm{~Hz}$. None of the arrival-time curves fit the entire frequency range of $2-256 \mathrm{~Hz}$ in our trials.

Dispersion characteristics of crack-waves recorded at a wellhead pressure of 3.0 MPa were also examined by fitting to ar- 


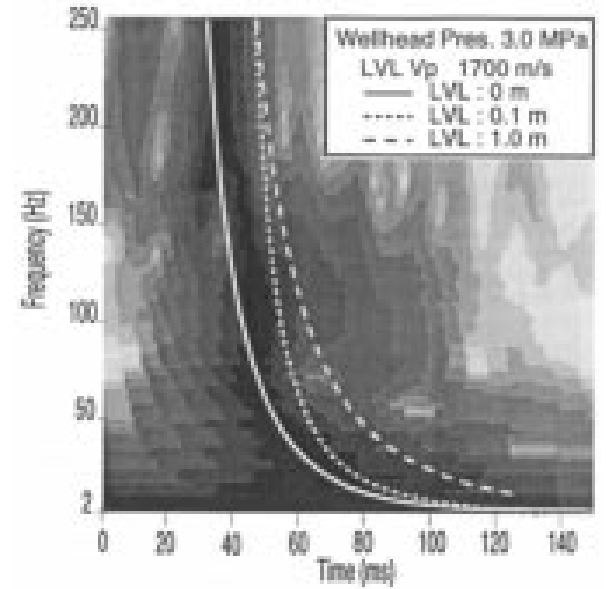

(a)

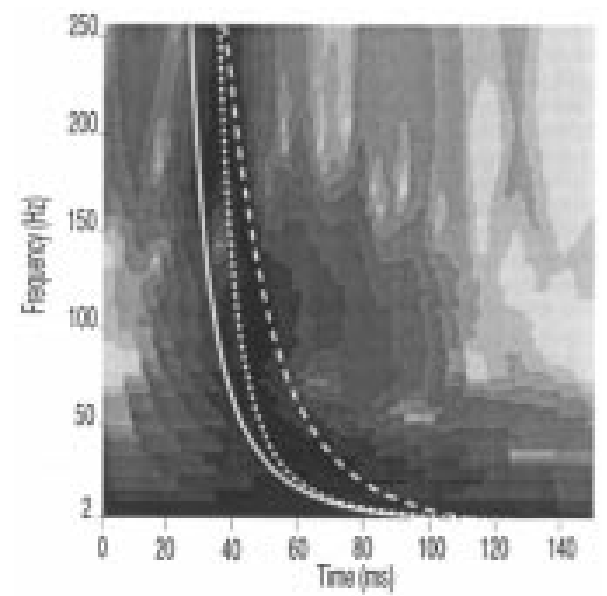

(b)

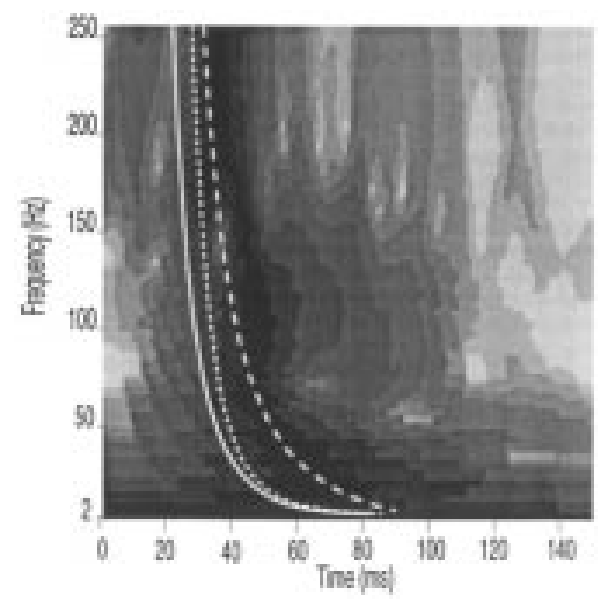

(c)

Fig. 11. Arrival times calculated with the LVL model and a time-frequency representation of crack-waves at a wellhead pressure of 3.0 $\mathrm{MPa}$. (a)-(c) The fluid layer is $0.5,1.0$, and $2.0 \mathrm{~mm}$ thick, respectively. Compressional and shear wave velocities are 1700 and $1020 \mathrm{~m} / \mathrm{s}$, respectively. Dispersion curves are calculated at LVL thicknesses of $0,0.1$, and $1.0 \mathrm{~m}$ in (a) and (b).

rival-time curves calculated with the LVL model. This wellhead pressure is higher than that in Fig. 10. A transmissibility test showed that the aperture in the artificial subsurface fracture increased at a wellhead pressure of $2.0 \mathrm{MPa}$ [13]. The velocity of a compressional wave of the LVL was $1700 \mathrm{~m} / \mathrm{s}$ at this wellhead pressure (Fig. 3). This velocity is lower than that at the begin- ning of water injection. Therefore, it is reasonable to assume that the geometry of the LVL varied due to the increase in wellhead pressure.

Fig. 11 shows some arrival-time curves for crack-waves recorded at a wellhead pressure of 3.0 $\mathrm{MPa}$. These crack-waves are more dispersive than those at a wellhead pressure of 0.4 $\mathrm{MPa}$. Differences between the dispersions are observed at frequencies below $70 \mathrm{~Hz}$. Differences between the arrival-time curves and the crack-waves measured at a wellhead pressure of 3.0 MPa are smaller than those at a wellhead pressure of 0.4 $\mathrm{MPa}$. However, none of the arrival-time curves showed a good fit over the entire frequency range of $2-256 \mathrm{~Hz}$. When the fluid layer is $2.0 \mathrm{~mm}$ and the LVL is $0.1 \mathrm{~m}$, the arrival-time curve shows a good fit with the onset of the relative maxima in our trials, even though the fit is not exact.

Differences between the arrival-time curves and the measured crack-waves decreased when the wellhead pressure increased. This means that the LVL model is more suitable for a highly pressurized subsurface fracture rather than for a closed subsurface fracture. However, the estimated thicknesses of the fluid layer and the LVL are not consistent with other data (i.e., the transmissibility test and crack-wave measurement). When the fluid layer is $2.0 \mathrm{~mm}$ and the LVL is $0.1 \mathrm{~m}$, the arrival-time curve showed a good fit to the crack-waves recorded at a wellhead pressure of 3.0 MPa in our trials. On the other hand, the thickness of the fluid layer was estimated to be $0.2 \mathrm{~mm}$ even at a wellhead pressure of 3.0 MPa in the transmissibility test [13]. The compressional wave velocity of rock is $3100 \mathrm{~m} / \mathrm{s}$ in this field and $1700 \mathrm{~m} / \mathrm{s}$ in the vicinity of the fracture at a wellhead pressure of 3.0 MPa. The air gun was suspended two meters above the intersection of a fracture, and the hydrophone was installed at another intersection. The estimated thickness $(0.1$ $\mathrm{m})$ of the LVL is too thin to decrease compressional wave velocity in the vicinity of the fracture. If the thickness of the LVL increases, the fluid layer should be thicker than $2.0 \mathrm{~mm}$ in the LVL model. However, we cannot agree with a fluid layer thicker than $2.0 \mathrm{~mm}$, since a fluid layer of $2.0 \mathrm{~mm}$ is ten times as thick as the result of the transmissibility test. Therefore, it is impossible to simulate arrival-time curves based solely on the LVL model. We should introduce mechanisms that increase the velocity of crack-waves in the LVL model.

\section{B. Arrival-Time Curves in the Crack-Stiffness Model}

Specific crack stiffness in a direction normal to the fluid layer and the thickness of the fluid layer were examined in the crackstiffness model. The physical properties of the bedrock layers and the fluid layer are the same as in the calculations for the LVL model. Fig. 12 shows arrival-time curves on a scalogram of crack-waves recorded at a wellhead pressure of $0.4 \mathrm{MPa}$. Combinations of crack stiffness and fluid-layer thickness were found by trial and error. Arrival-time curves for a high crack stiffness show weak dispersion. They fit the relative maxima in the scalogram better than those in the LVL model. The best combination in our trials was a crack stiffness of $90 \mathrm{GPa} / \mathrm{m}$ and a fluid layer thickness of $0.2 \mathrm{~mm}$.

Calculated arrival-time curves and crack-waves, recorded at a wellhead pressure of 3.0 MPa, are compared in Fig. 13. The crack-waves at a wellhead pressure of 3.0 MPa were more 


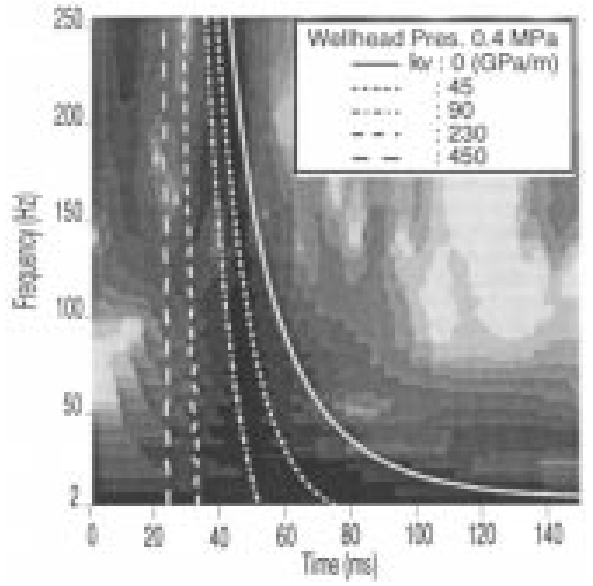

(a)

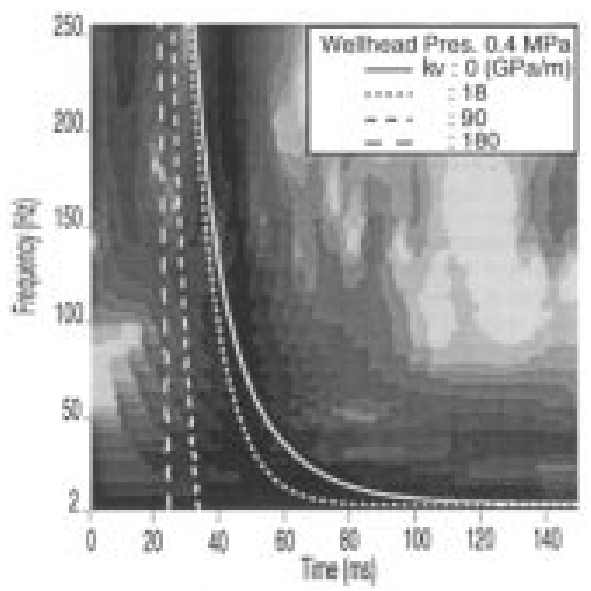

(b)

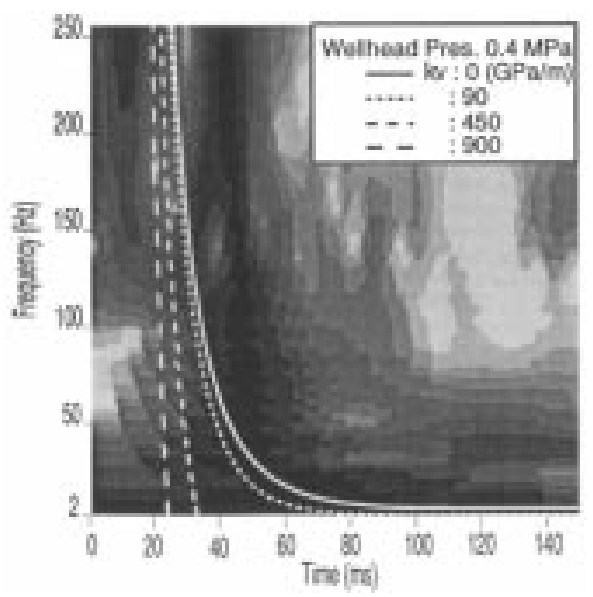

(c)

Fig. 12. Arrival times calculated with the crack-stiffness model and a time-frequency representation of the crack-waves at the beginning of water injection. (a)-(c) The fluid layer is $0.2,0.5$, and $1.0 \mathrm{~mm}$ thick, respectively. The arrival times of crack-waves were calculated at specific levels of crack stiffness.

strongly dispersive than those at a wellhead pressure of $0.4 \mathrm{MPa}$. When the crack stiffness is $18 \mathrm{GPa} / \mathrm{m}$ and the thickness of the fluid layer is $0.5 \mathrm{~mm}$, the arrival-time curve shows a good fit to the relative maxima in the scalogram for crack-waves at a wellhead pressure of 3.0 MPa in our trials.

The estimations of crack stiffness and the thickness of the fluid layer are consistent with the opening of the fracture. When

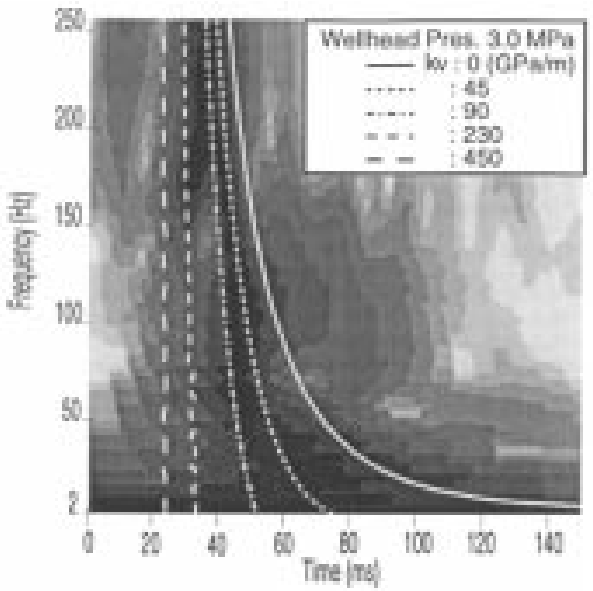

(a)

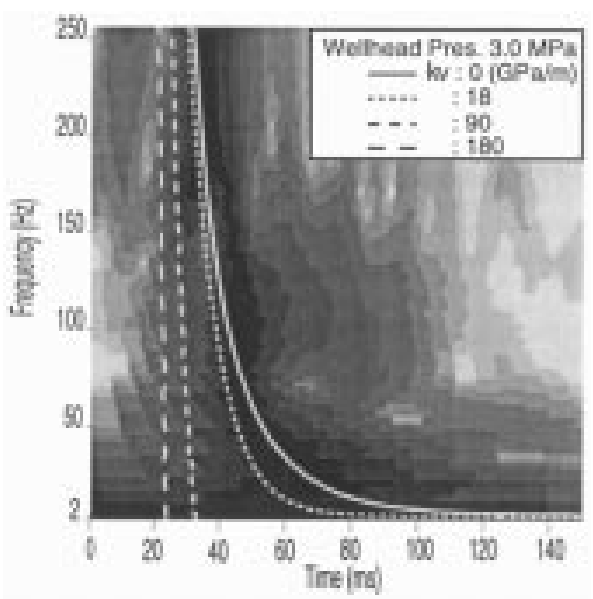

(b)

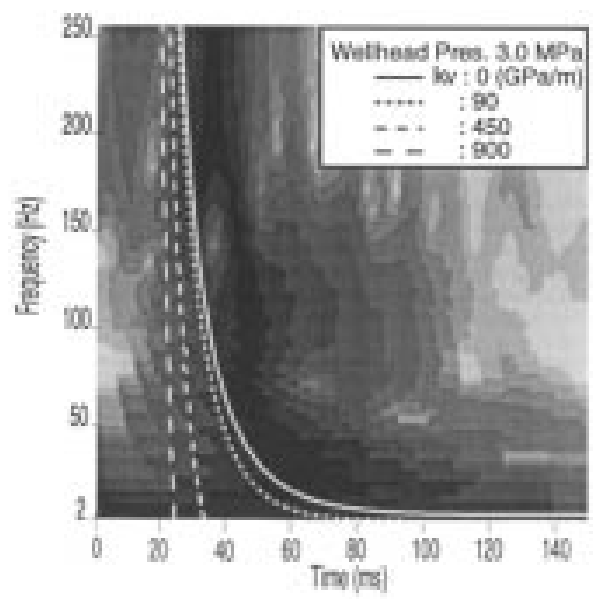

(c)

Fig. 13. Arrival times calculated with the crack-stiffness model and a time-frequency representation of crack-waves at a wellhead pressure of 3.0 MPa. (a)-(c) The fluid layer is $0.2,0.5$, and $1.0 \mathrm{~mm}$ thick, respectively. The arrival times of crack-waves were calculated at specific levels of crack stiffness.

the wellhead pressure increased, the thickness of the fluid layer increased from 0.2 to $0.5 \mathrm{~mm}$, and the crack stiffness decreased from 90 to $18 \mathrm{GPa} / \mathrm{m}$. In a simple model of a subsurface fracture shown in Fig. 14, stiffness per unit area in the direction normal to the interface is

$$
k_{v}=\frac{S}{\Delta h}=\frac{E}{h} \frac{A_{c}}{A}
$$




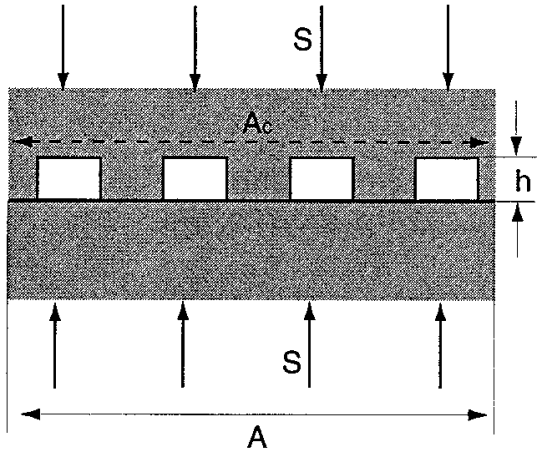

Fig. 14. Simple crack model.

where $S$ is the compressional stress normal to the interface, $A$ is the entire area, $A_{c}$ is the contact area, and $E$ is Young's modulus. The decrease in crack stiffness, which was observed in the crack-wave measurement, can be explained by an increase in the aperture of the fracture and by a decrease in the contact area between asperities on crack surfaces, or both.

\section{CONCLUSIONS}

We have investigated the dispersions of crack-waves using two crack models. We analyzed crack-waves that were measured in an artificial subsurface fracture at a depth of about 370 $\mathrm{m}$. The WT of the crack-waves measured at an artificial subsurface fracture showed positive dispersion. The crack-waves were more strongly dispersive at a wellhead pressure of $3.0 \mathrm{MPa}$ than at a wellhead pressure of 0.4 MPa. The LVL model and the crack-stiffness model were used to calculate arrival-time curves of crack-waves. The arrival-time curves calculated with the LVL model were late relative to the measured crack-waves. On the other hand, the curves calculated with the crack-stiffness model agreed with the dispersions of the crack-waves. We determined the best combinations of the thickness of the fluid layer and the crack stiffness at two levels of wellhead pressure (0.4 and 3.0 $\mathrm{MPa})$. These estimates with the crack-stiffness model are consistent with opening of the fracture.

We noted that contact and the low-velocity zone are important physical properties for estimating subsurface fractures. Contact is expressed in the crack-stiffness model. On the other hand, the LVL model represents a low-velocity zone. The results of the crack-wave dispersion indicate that contact is more dominant than the low-velocity zone in physical properties that affect crack-wave dispersion.

\section{REFERENCES}

[1] B. Chouet, "Dynamics of a fluid-driven crack in three dimensions by the finite difference method," J. Geophys. Res., vol. 91, pp. 13967-13992, Dec. 1986.

[2] Y. G. Li, P. C. Leary, and K. Aki, "Observation and modeling of fault-zone fracture seismic anisotropy-II: P-wave polarization anomalies," Geophys. J. R. Astron. Soc., vol. 91, pp. 485-492, 1987.

[3] M. Lou, J. A. Rial, and P. E. Malin, "Modeling fault-zone guided waves of microearthquakes in a geothermal reservoir," Geophysics, vol. 62, pp. 1278-1284, July/Aug. 1997.

[4] K. Nagano, H. Saito, and H. Niitsuma, "Guided waves trapped in an artificial subsurface fracture," Geotherm. Sci. Technol., vol. 5, no. 1/2, pp. 63-70, 1995.
[5] K. Nagano and H. Niitsuma, "Crack stiffness from crack wave velocities," Geophys. Res. Lett., vol. 23, pp. 689-692, Mar. 1996.

[6] K. Nagano, K. Sato, and H. Niitsuma, "Polarization of crack waves along an artificial subsurface fracture," Geophys. Res. Lett., vol. 23, pp. 2017-2020, Aug. 1996.

[7] K. Nagano, "Crack-wave dispersion at a fluid-filled fracture with lowvelocity layers," Geophys. J. Int., vol. 134, pp. 903-710, Sept. 1998.

[8] K. Hayashi and K. Sato, "A theoretical study of AE traveling through a fluid-filled crack with application to characterization of a geothermal reservoir crack," in Progress in Acoustic Emission VI, T. Kishi, K. Takahashi, and M. Ohtsu, Eds. Tokyo, Japan: Japanese Soc. NDI, 1992, pp. 423-430.

[9] H. Niitsuma and H. Saito, "Characterization of a subsurface artificial fracture by the triaxial shear shadow method," Geophys. J. Int., vol. 107, pp. 485-491, Nov. 1991.

[10] K. Tanaka, H. Moriya, H. Asanuma, and H. Niitsuma, "Detection of travel time delay caused by inflation of single artificial fracture," Geotherm. Sci. Technol., to be published.

[11] V. Ferrazzini and K. Aki, "Slow waves trapped in a fluid-filled infinite crack: Implication for volcanic tremor," J. Geophys. Res., vol. 92, pp. 9215-9223, Aug. 1987.

[12] H. Niitsuma, "Fracture mechanics design and development of HDR reservoirs-concept and results of the $\Gamma$-project, Tohoku University, Japan," Int. J. Rock Mech. Min. Sci. Geomech. Abstr, vol. 26, no. 3/4, pp. $169-175,1989$.

[13] K. Hayashi and H. Abé, "Evaluation of hydraulic properties of the artificial subsurface system in Higashihachimantai geothermal model field," J. Geotherm. Res. Soc. Jpn., vol. 11, no. 3, pp. 203-215, 1989.

[14] F. Hlawatsh and G. F. Boudreaux-Bartels, "Linear and quadratic timefrequency signal representations," IEEE Signal Processing Mag., vol. 9, pp. 21-67, Apr. 1992.

[15] S. Qian and D. Chen, Joint Time-Frequency Analysis, Methods and Applications. Englewood Cliffs, NJ: Prentice-Hall, 1996.

[16] L. J. Pyrak-Nolte and D. D. Nolte, "Wavelet analysis of velocity dispersion of elastic interface waves propagating along a fracture," Geophys. Res. Lett., vol. 22, pp. 1329-1332, June 1995.

[17] R. Kronland, J. Morlet, and A. Grossmann, "Analysis of sound patterns through wavelet transforms," Int. J. Pattern Recognit. Artif. Intell., vol. 1, no. 2, pp. 273-302, 1987.

[18] N. A. Haskell, "The dispersion of surface waves on multilayered media," Bull. Seismol. Soc. Amer, vol. 43, pp. 17-34, 1953.

[19] K. Hayashi, T. Ito, and H. Abé, "In situ stress determination by hydraulic fracturing-A method employing an artificial notch," Int. J. Rock Mech. Min. Sci. Geomech. Abstr., vol. 26, no. 3/4, pp. 197-202, 1989.

Koji Nagano received the B.Eng., M.Eng., and the Dr.Eng. degrees in electrical engineering from Tohoku University, Sendai, Japan, in 1983, 1986, and 1989, respectively.

From 1989 to 1991, he was a Fellow of the Japan Society for the Promotion of Science for Japanese Junior Scientists. He was a Research Associate at the Department of Resources Engineering, Tohoku University from 1991 to 1992, and then joined the Department of Computer Science and Systems Engineering, Muroran Institute of Technology, Muroran, Japan, where he is currently a Research Associate. His research interests are seismic signal processing, cross-hole seismic measurement, and wave propagation for characterization of subsurface crack.

Hiroaki Niitsuma received the Ph.D. degree from Tohoku University, Sendai, Japan, in 1975.

He has been a Professor with the Department of Geoscience and Technology, Faculty of Engineering, Graduate School of Tohoku University since 1988. His recent research interests are in multicomponent seismic measurement and signal processing, bore hole measurement, and characterization of geothermal reservoir. He is a Representative of the International Collaboration Program "Establishment of new mapping/imaging technologies for advanced energy extraction from deep geothermal reservoirs (MTC project)," funded by NEDO and MESC. He was Chairman of the Subsurface Instrumentation Division of MMIJ from 1989 to 1995 . He is now a Director of SEGJ, MMIJ, the SPWLA Japan Chapter, and a Councilor of the Geothermal Research Society of Japan. 